\title{
Dynamics of porous saturated media, checking of the generalized law of Darcy
}

\author{
Jean-Louis Auriault, Lionel Borne, and Rene Chambon \\ Institut de Mécanique de Grenoble, U.A. 06 C.N.R.S., B.P. 68, 38402 Saint Martin D'Heres Cedex, France
}

The homogenization process applied to fine periodic deformable saturated porous medium under dynamic solicitations leads to the macroscopic description. This method enables us to perform a complete calculation of the effective parameters. The main fact is that the formulation so obtained-similar to Biot's results-exhibits a generalized example of Darcy's law which contains all the dynamic couplings between the two phases. After recalling the main facts of the subject this work presents some properties of the generalized Darcy coefficient and an experimental checking. An agreement between experimental and numerical results using the homogenization process is obtained.

\section{INTRODUCTION}

The macroscopic description of fluid-filled porous media was first considered by Biot' directly at the macroscopic level for quasistatics, as well as for problems of dynamics. The formulation so obtained is now generally accepted by scientists in this field. Plona's experimental setup, ${ }^{2}$ which exhibits a second bulk compressional wave, has given more consistency to this theory (see also Ref. 3). However, no complete test could be obtained since Biot's phenomenological approach does not lead to numerical values for the effective coefficients involved in the equations describing the macroscopic behavior. This consideration has led to truncated formulations used by many authors. ${ }^{416}$ Other approaches, among which are mixture theories, give still more complicated possiblilities for checking. ${ }^{17-19}$

On the contrary, the homogenization process, which constructs the macroscopic description from the microscopic one, enables the computation of effective coefficients when in full use, i.e., when the pore structure is periodic (see Refs. 20-23, and for a review on this subject see Ref. 24). For the problem under consideration, the "macroscopic dynamic" so obtained is similar to that of Biot's theory, but it points out a generalized Darcy coefficient entering a generalized Darcy law and clustering all the information about the dynamic coupling between the solid and the fluid. More than this simplification, the process leads to local problems. Variational formulations well adapted to the finite element computation techniques also lead to the effective parameters, which are deduced by averaging operations. Until now, few attempts at experimental checking have been performed following this process. ${ }^{25.26}$

The purpose of this paper is to study this generalized Darcy coefficient (in fact its inverse $H$ with a clearer physical meaning) and to present an experimental checking.

In Sec. I the principal results of the homogenization process for fluid-filled porous media are recalled, first when the skeleton is rigid and second when it is deformable. Then considerations in relation with Biot's formulation are given.

Section II is devoted to the asymptotic monochromatic behavior of coefficient $H(w)$ when the pulsation $w$ increases to infinity, in order to supply the ill-conditioned computational problem when the frequencies are of high value.

In Sec. III an experimental setup is presented. The results are compared with the numerical computation of the corresponding coefficient $H(w)$.

\section{HOMOGENIZATION PROCESS APPLIED TO POROUS SATURATED MEDIA}

The homogenization technique can be considered when, for the medium, the microscopic and the macroscopic scales, of characteristic lengths $l$ and $L$, respectively, are of different orders of magnitude. The problem with one particular homogenization method was investigated: the socalled double-scale method described in Refs. 27 and 28 . A small dimensionless parameter $\epsilon=l / L$ and two dependent spatial variables $x$ and $y=x / \epsilon$ are used, which describe the macroscopic and microscopic situations, respectively. Moreover, the pore structure is assumed to be periodic with a period $\Omega$ (for the variable $y$ ), which implies the periodicity for the geometry and the local parameters.

Let us consider a macroscopic domain $D$ of size $L$, consisting of a large number $\epsilon^{-1}$ of periods. It was required that the behavior with respect to $x$ should be described, i.e., an homogenized continuous situation where the heterogeneities at the pore level are smoothed. A quantity $v$ related to this medium is, in fact, dependent on the variable $\mathbf{x}$ and on the small heterogeneities, i.e., $\epsilon$. The homogenized situation is described by the asymptotic value of $v$ when $\epsilon$ vanishes, even when the zero value is not effectively reached in a concrete case. In the problem under consideration, for instance, the pore size is not zero-valued. Moreover, the method leads to a first approximation for the macroscopic equivalent be- 
havior. The heuristic method consists of seeking the unknown in the form

$$
\begin{aligned}
v & =v_{\epsilon}(\mathbf{x})=v\left(\mathbf{x}, \mathbf{x} \epsilon^{-1}\right)=v(\mathbf{x}, \mathbf{y}) \\
& =v^{(0)}(\mathbf{x}, \mathbf{y})+\epsilon v^{(1)}(\mathbf{x}, \mathbf{y})+, \ldots,
\end{aligned}
$$

where the $v^{(i)}$ are $\Omega$ periodic, by introducing such asymptotic developments into the equations describing the local behavior. Then the powers of $\epsilon$ are identified. (Boldfaced symbols are used for tensor quantities.) In the following section, the case of a rigid skeleton is presented. Detailed developments are available in Ref. 24.

\section{A. Dynamics of fluid flow through a rigid porous medium}

In Fig. 1, a sketch view of two periods of a porous medium (two-dimensional case) is presented. The fluid is assumed to be Newtonian and incompressible (the second hypothesis can be removed; refer to the end of this section). It undergoes a small perturbation around the rest. The velocity $\nabla$ is small enough to satisfy the linearized Navier-Stokes equations. On the contrary, the linear dynamical term is taken into account and the transient Reynold's number is of the order of 1:

$$
R_{R}=l^{2} \omega \rho \mu^{-1}=O(1) .
$$

$\rho$ denotes the mass per unit volume and $\mu$ is the dynamic viscosity. Thus the set of equations for a monochromatic isothermic perturbation, at the microscopic level, is of the form

$$
\begin{aligned}
& \mu \Delta \nabla=-\nabla p+i \omega \rho \mathbf{v}, \\
& \nabla \nabla=0, \\
& \left.\nabla\right|_{\Gamma}=0 .
\end{aligned}
$$

$p$ is the increment of pressure (positive in traction) due to the perturbation, $\Delta$ is the Laplacianoperator and $\nabla p$ denotes the gradient of $p$. In order to verify relation (2) let

$$
\boldsymbol{\mu}=\boldsymbol{\mu}^{\prime} \epsilon^{2} \text {. }
$$

Therefore Eq. (3) on the rescaled form appears as

$$
\mu^{\prime} \epsilon^{2} \Delta \nabla=-\nabla p+i \omega \rho \nabla .
$$

Other rescaled equations still meeting the relation expressed in Eq. (2) are possible, for example,

$$
\mu^{\prime} \epsilon \Delta \nabla=-\nabla p+i \omega \rho \epsilon^{-1} \mathbf{v}
$$

Equations of this type lead to the same result in the case of a rigid skeleton.

Such developments as in Eq. (1) for $\mathbf{v}$ and $p$ are introduced in Eqs. (4)-(6). Remember that the classical operator $d / d x$ now becomes

$$
\frac{\partial}{\partial x}+\epsilon^{-1} \frac{\partial}{\partial y}
$$

since the variables $x$ and $y$ are dependent. Equation (6) at $\epsilon^{-1}$ order gives

$$
\nabla_{y} p^{(0)}=0,
$$

where the subscript $y$ denotes a derivation with respect to $y$. It follows that

$$
p^{(0)}=p^{(0)}(\mathbf{x}) \text {. }
$$

Equation (6) at $\epsilon^{0}$, Eq. (4) at $\epsilon^{-1}$, and Eq. (5) at $\epsilon^{0}$ orders give

$$
\begin{aligned}
& \mu^{\prime} \Delta_{y} \nabla^{(0)}=-\nabla_{x} p^{(0)}-\nabla_{y} p^{(1)}+i \omega \rho \nabla^{(0)}, \\
& \nabla_{y} \nabla^{(0)}=0, \\
& \left.\nabla^{(0)}\right|_{\Gamma}=0,
\end{aligned}
$$

$\nabla^{(0)}$, and $p^{(1)} \Omega$ périodic. This is a so-called cell problem of the unknowns $\nabla^{(0)}$ and $p^{(1)}$.

Considering the space $\mathscr{W}$ of $\Omega$-periodic, free-divergence, and complex-valued vectors with a zero value on $\Gamma$, the variational equivalent formulation is written as follows ${ }^{24}$ :

$\forall \alpha \in \mathscr{W}$,

$\int_{\Omega_{l}}\left(\mu^{\prime} \frac{\partial v_{k}^{(0)}}{\partial y_{j}} \frac{\partial \widetilde{\alpha}_{k}}{\partial y_{j}}+i \omega \rho v_{k}^{(0)} \widetilde{\alpha}_{k}\right) d \Omega=\int_{\Omega_{l}} \frac{d p^{(0)}}{d x_{i}} \widetilde{\alpha}_{i} d \Omega$,

where $\widetilde{\alpha}$ is the complex conjugate of $\alpha$. This is a well-posed problem with existence and uniqueness. The linearity carries forward to the solution

$$
\nabla^{(0)}=\mathbf{k}(\mathbf{y}, \omega) \nabla_{x} p^{(0)}
$$

where the $k_{i j}$ are particular solutions of Eq. (10). The secondorder tensor $k$ is $\omega$-dependent and complex-valued. Equation (4) at the $\epsilon^{(0)}$ order gives

$$
\nabla_{y} \nabla^{(1)}+\nabla_{x} \nabla^{(0)}=0
$$

Integrating over $\Omega_{l}$ and taking into account the periodicity of $\nabla^{(1)}$ and the adherence condition, Eq. (5) at $\epsilon$ order, is obtained at the first order:

$$
\begin{aligned}
& \boldsymbol{\nabla}_{x}\langle\boldsymbol{\nabla}\rangle=0, \quad\langle\boldsymbol{\nabla}\rangle=|\boldsymbol{\Omega}|^{-1} \int_{\Omega_{l}} \mathbf{\nabla} d \Omega, \\
& \langle\boldsymbol{\nabla}\rangle=\mathbf{K} \boldsymbol{\nabla}_{x} p,
\end{aligned}
$$

where $\mathbf{K}, \omega$-dependent with a complex value, is a generalized Darcy coefficient. Equations (11) and (12) stand for the macroscopic behavior. When a transient motion is considered the right-hand side of Eq. (12) becomes a convolution product between the inverse Fourier transforms of $K$ and $\nabla_{x} p$. The fundamental properties for $\mathbf{K}$ deduced from this approach can be recalled.

(1) $\mathbf{K}$ goes to the classical quasistatic Darcy coefficient as $w$ vanishes.

(2) $\mathbf{K}$ is symmetric. (The reciprocal Onsager relations are not necessary for this.)

(3) $\langle\nabla\rangle$, which is a volume mean value, is also a flux, i.e., a Darcy velocity.

(4) Let $\mathbf{H}=\mathbf{H}_{1}+\imath \mathbf{H}_{2}=\mathbf{K}^{-1}$. Equation (11) becomes the momentum balance

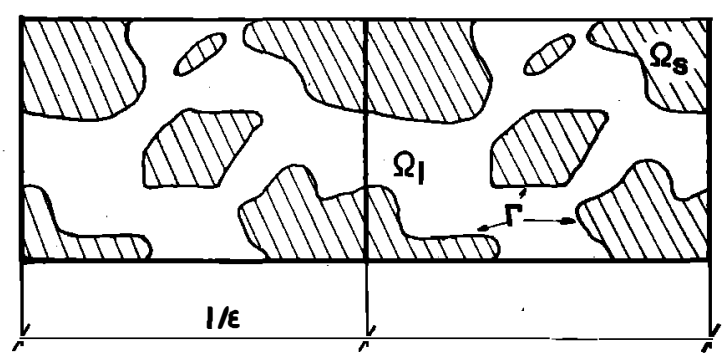

FIG. 1. Sketch of two periods of a porous medium. $\boldsymbol{\Omega}_{\boldsymbol{l}}=$ liquid part, $\boldsymbol{\Omega}_{s}=$ solid part, $\Gamma=$ contact surface. 


$$
\nabla_{x} \rho=H_{1}\langle\nabla\rangle+H_{2} \omega^{-1}\langle\dot{\nabla}\rangle .
$$

The real part $\mathbf{H}_{1}$ of $\mathrm{K}^{-1}$ is dissipative, the imaginary part $\mathbf{H}_{2}$ is an inertial part, and it stands

$$
n \omega^{-1} H_{2}>\rho
$$

in the isotropic case ( $n$ denotes the porosity). If the fluid is compressible, only Eq. (11) is modified. The continuity equation [Eq. (4)] becomes

$$
\nabla \rho \nabla+i \omega \bar{\rho}=0
$$

and

$$
p=K_{\omega} \nabla \nabla / i \omega,
$$

where $\rho$ is the mass per unit volume at rest, $\bar{\rho}$ is its increment under the perturbation, and $K_{\omega}$ is the bulk modulus of the fluid. At $\epsilon^{-1}$ order this leads to Eq. (8): $\nabla_{y} \nabla^{(0)}=0$, and Eqs. (7) $(9)$ are still valid since at $\epsilon^{0}$ order the new isotropic viscous term disappears from Navier-Stokes equations. So, the generalized Darcy law [Eq. (12)] remains unchanged. On the contrary, the continuity balance equation at $\epsilon^{0}$ order gives, using for $\bar{\rho}$ an asymptotic expansion such as in Eq. (1):

$$
\nabla_{y}\left(\rho \nabla^{(1)}\right)+\nabla_{x}\left(\rho \nabla^{(0)}\right)=-i \omega \rho^{(0)}=i \omega p^{(0)} \rho / K_{\omega} .
$$

As previously shown, the volume mean value process is carried out, which leads to a different equation from Eq. (11):

$$
\nabla_{x}\langle\nabla\rangle=n i \omega p / K_{\omega} .
$$

For the cases of a cylindrical slit or a circular cylinder, analytical results are available. The method gives, for a slit of thickness $2 a$,

$$
\begin{aligned}
K= & n(i \omega \rho)^{-1}\left\{1-a^{-1}(v / i \omega)^{1 / 2}\right. \\
& \left.\times \tanh \left[(i \omega / v)^{1 / 2} a\right]\right\}, \quad v=\mu \rho^{-1},
\end{aligned}
$$

and for a cylinder of diameter $2 a$,

$$
K=-n(i \omega \rho)^{-1} J_{2}\left[i a(i \omega / v)^{1 / 2}\right]\left\{J_{0}\left[i a(i \omega / v)^{1 / 2}\right]\right\}^{-1} .
$$

$J_{2}$ and $J_{0}$ are Bessel functions. This last result was used in Refs. 25 and 26 for an experimental control on a damper. It is of interest to point out that the problem of cylindrical ducts can be solved directly from Navier-Stokes equations without the help of the homogenization method. This comes from the arbitrary periodicity along the axes of the ducts: The direct boundary problem at the macroscopic level is identical to the local boundary problem over the period introduced by the homogenization process. So, the abovementioned results for a circular cylinder or a slit are classical. For more sophisticated geometries, which will be investigated, no direct solution is possible since the computational problem becomes more and more complicated and ill conditioned as the number of periods is increasing. Moreover, the direct solution does not provide the equivalent macroscopic description of the medium. In this case the homogenization process is necessary.

\section{B. Dynamics of a fluid-saturated deformable porous medlum 24}

The skeleton is now assumed to obey an elastic equation and the stresses in the solid and the liquid are of the same order. For the liquid, the assumptions of Sec. I A are still valid. Particularly, the rescaled Eq. (6) can be admitted [see remark (3) of Sec. I C]. Then, after performing the homog- enization process, the set of equations for the macroscopic motion with a constant pulsation is written (the stresses are positive in traction) as follows:

$$
\begin{aligned}
& \nabla_{x}\left\langle\sigma^{T}\right\rangle=\left\langle\rho_{s}\right) \ddot{\mathbf{u}}_{s}+\rho\langle\dot{v}\rangle, \\
& \left\langle\sigma^{T}\right\rangle=\operatorname{ce}_{x}\left(\mathbf{u}_{s}\right)+\alpha p, \\
& \langle\nabla\rangle-n \dot{\mathbf{u}}_{s}=\mathbf{K}\left(\nabla_{x} p-\rho \ddot{u}_{s}\right), \\
& \nabla_{x}\left[\mathbf{K}\left(\nabla_{x} p-\rho \ddot{u}_{s}\right)\right]=-\alpha \dot{e}_{x}\left(\mathbf{u}_{s}\right)+\beta \dot{p},
\end{aligned}
$$

where $\sigma^{T}$ is the total stress defined by

$$
\begin{aligned}
& \boldsymbol{\sigma}^{T}= \begin{cases}\sigma_{s}, & \text { the stress in the skeleton, } \\
\sigma_{l}, & \text { the stress in the liquid, }\end{cases} \\
& \left\langle\sigma^{T}\right\rangle=|\Omega|^{-1} \int_{\Omega} \sigma^{T} d \Omega,
\end{aligned}
$$

$\rho_{s}$ is the mass per unit volume in the solid, $u_{s}$ is the displacement in the solid, $\mathbf{e}$ is the deformation, and $\mathbf{c}$ is the elastic tensor. The effective parameters $\mathbf{c}, \alpha, \mathbf{K}$, and $\beta$ can be computed.

The tensor $\mathbf{K}$ is identical to that defined in Sec. I A for a rigid skeleton. Introducing the partial stress $\left\langle\sigma_{s}\right\rangle$ in the solid by

$$
\left\langle\sigma^{T}\right\rangle=\left\langle\sigma_{s}\right\rangle+n p \mathbf{I}, \quad \mathbf{I} \text { unit tensor, }
$$

a formulation similar to Biot's was obtained:

$$
\begin{aligned}
& \nabla_{x}\left\langle\sigma_{s}\right\rangle=\mathbf{b}\left(\dot{\mathbf{u}}_{s}-\dot{\mathbf{u}}_{l}\right)+\boldsymbol{p}_{11} \ddot{\mathbf{u}}_{s}+\boldsymbol{\rho}_{12} \ddot{\mathbf{u}}_{l}, \\
& \nabla_{x} n p=-\mathbf{b}\left(\dot{\mathbf{u}}_{s}-\dot{\mathbf{u}}_{l}\right)+\boldsymbol{p}_{22} \ddot{\mathbf{u}}_{l}+\boldsymbol{p}_{21} \ddot{\mathbf{u}}_{s}, \\
& \left\langle\sigma_{s}\right\rangle=\mathbf{Q} \theta+\operatorname{de}_{x}\left(\mathbf{u}_{s}\right), \\
& n p=\mathbf{Q} \mathbf{e}_{x}\left(\mathbf{u}_{s}\right)+\boldsymbol{R} \theta,
\end{aligned}
$$

with

$$
\begin{aligned}
& \mathbf{Q}=n(\alpha-n \mathrm{I}) / \beta, \quad R=n^{2} / \beta, \\
& \mathbf{b}=n^{2} \mathbf{H}_{1}(\omega), \\
& \boldsymbol{\rho}_{11}=\left(\rho_{s}\right) \mathrm{I}-\boldsymbol{\rho}_{21}, \quad \boldsymbol{\rho}_{22}=n^{2} \mathbf{H}_{2}(\omega) \omega^{-1}, \\
& \boldsymbol{\rho}_{12}=\boldsymbol{\rho}_{21}=n \rho \mathbf{I}-n^{2} \mathbf{H}_{2}(\omega) / \omega .
\end{aligned}
$$

From the relation in Eq. (13), in the isotropic case,

$$
\begin{aligned}
& \rho_{11}>\left\langle\rho_{s}\right\rangle, \quad \rho_{12}=\rho_{21}<0, \\
& \rho_{22}>n \rho
\end{aligned}
$$

was obtained.

\section{Remarks}

(1) The main fact to be pointed out is the simplicity of the description in which a unique parameter $\mathbf{H}=\mathrm{K}^{-1}$ involves the whole dynamic coupling between the liquid and the solid.

(2) The physical meaning of macroscopic quantities is preserved, as $\langle\nabla\rangle,\left\langle\sigma^{T}\right\rangle$, or $\left\langle\sigma_{s}\right\rangle$, defined as volume mean values, are fluxes and then are Cauchy stresses.

(3) Unlike the case investigated in Sec. I A, rescaled equations other than Eq. (6) lead to different macroscopic behaviors. In view of obtaining a Biot's description, the two necessary assumptions are relation $(2): l^{2} \omega \rho \mu^{-1}=O(1)$ and $p l \epsilon \mu^{-1}|v|^{-1}=O(1)$, which means that the viscous stress in the liquid is small $[O(\epsilon)]$ compared to the pressure. See Ref. 24 for different situations. 


\section{THE GENERALIZED DARCY COEFFICIENT AT HIGH FREQUENCIES}

As the pulsation $\omega$ is increasing, the numerical problem associated with the variational formulation, Eq. (10), becomes more and more ill conditioned. Therefore, a study of $\mathbf{K}(\omega)$ as $\omega \rightarrow \infty$ is useful, ${ }^{29}$ even if, for a large $\omega$, the macroscopic description presented in Sec. I is no longer valid. As $\omega$ is increasing, the wavelength decreases and becomes $O(l)$; the $\Omega$ periodicity is no longer available for the velocity $v$ and the homogenization process as described above is not heuristic. Scattering appears.

The set of Eqs. (7) $(9)$ when $\omega$ is large are considered. Let us assume $v^{(0)}$ and $p^{(1)}$ to be of the form

$$
\begin{aligned}
& \nabla^{(0)}=\nabla_{(0)}+\eta \nabla_{(1)}+\cdots, \\
& p^{(1)}=p_{(0)}+\eta p_{(1)}+\cdots,
\end{aligned}
$$

where $\eta=\omega^{-1}<1$ and $\nabla_{i}, p_{i} \Omega$ periodic. At lower orders

$$
i \rho \nabla_{(0)}=0, \quad \nabla_{y} \nabla_{(0)}=0,\left.\quad \nabla_{0}\right|_{\Gamma}=0
$$

is obtained, and obviously $\nabla_{(0)}=0$. Then

$$
\begin{aligned}
& \nabla_{x} p^{(0)}+\nabla_{y} p_{(0)}-i \omega \rho \nabla_{(1)}=0, \\
& \nabla_{y} \nabla_{(1)}=0, \\
& \left.\nabla_{(1)}\right|_{\Gamma}=0
\end{aligned}
$$

arrives. From Eq. (14) there is rot $\nabla_{(1)}=0$. This system is not well posed: Eqs. (14) and (15) describe an incompressible and irrotational perfect fluid with the body forces, $\nabla_{x} p^{(0)}$, not compatible with the adherence equation (16). The problem can be classically solved by considering first Eqs. (14) and (15) with an impermeability condition on $\Gamma$ and second, introducing a viscous boundary layer along $\Gamma$, matching the perfect fluid solution with the adherence condition. The perfect fluid will give a first approximation for $\mathbf{H}$ with respect to $\omega^{-1}$. Since the viscosity is zero at this initial step, the first approximation for $\nabla_{(1)}$ will only concern the inertial part $\mathbf{H}_{2}$ of $\mathbf{H}$. In this case all $\nabla_{(1)}$ for $i \geqslant 2$ are zero valued. A secondorder approximation will be introduced by the boundary layer.

The velocity $\nabla_{p}$ and the pressure $p_{p}$ in the perfect fluid are defined by

$$
\begin{aligned}
& \nabla_{x} p^{(0)}+\nabla_{y} p_{p}-i \omega \rho \nabla_{p}=0, \\
& \nabla_{y} \nabla_{p}=0,\left.\quad \nabla_{p} \cdot \mathbf{N}\right|_{\Gamma}=0,
\end{aligned}
$$

where $\mathbf{N}$ is the unit external normal of $\Omega_{l}$ to $\Gamma$ and $\nabla_{p}, p_{p}$ are periodic. Note that rot $\nabla_{p}=0$. The velocity potential $\phi$ is introduced by $\nabla_{p}=\operatorname{grad} \phi$ and the problem is the determination of $\phi$ with

$$
\begin{aligned}
& \Delta_{y} \phi=0, \\
& \left.\nabla_{y} \phi \cdot \mathbf{N}\right|_{\Gamma}=0,
\end{aligned}
$$

where $\nabla_{y} \phi$ is $\Omega$-periodic, but not necessary $\phi$. So a solution of the form

$$
\phi=\alpha+A_{i} y_{i}
$$

is sought, where $\mathbf{A}$ is a constant vector to be specified forward and $\alpha$ is a real scalar field, $\Omega$-periodic with $\int_{\Omega_{1}} \alpha d \Omega=0$. (Since $\phi$ is defined to an arbitrary additive constant, this external condition is not restrictive.)
The space $E$ of real scalar functions $\alpha$, defined over $\Omega_{l}$, $\boldsymbol{\Omega}$-periodic, with a zero-mean value and the scalar product, is introduced:

$$
(\alpha, \beta)=\int_{\Omega_{t}} \frac{\partial \alpha}{\partial y_{j}} \frac{\partial \beta}{\partial y_{j}} d \Omega .
$$

Multiplying Eq. (18) by $\beta \in E$, integrating over $\Omega_{l}$, using the divergence theorem, the $\Omega$ periodicity, and Eq. (19), we obtain:

$$
\begin{aligned}
\forall \beta \in E, \quad 0 & =\int_{\Omega_{t}} \beta \Delta_{y} \phi d \Omega \\
& =\int_{\Omega_{t}}\left[\frac{\partial}{\partial y_{j}}\left(\beta \frac{\partial \phi}{\partial y_{j}}\right)-\frac{\partial \beta}{\partial y_{j}} \frac{\partial \phi}{\partial y_{j}}\right] d \Omega \\
& =\int_{\delta \Omega_{t}} \beta \frac{\partial \phi}{\partial y_{j}} N_{j} d s-\int_{\Omega_{i}} \frac{\partial \beta}{\partial y_{j}} \frac{\partial \phi}{\partial y_{j}} d \Omega \\
& =-\int_{\Omega_{l}} \frac{\partial \beta}{\partial y_{j}} \frac{\partial \phi}{\partial y_{j}} d \Omega, \\
\int_{\Omega_{t}} \frac{\partial \alpha}{\partial y_{j}} \frac{\partial \beta}{\partial y_{j}} d \Omega & =-\int_{\Omega_{i}} A_{i} \frac{\partial \beta}{\partial y_{t}} d \Omega .
\end{aligned}
$$

Equation (20) represents a variational relation, equivalent to Eqs. (17) and (18). The solution is unique and because of the linearity, may be written as $\alpha=t_{i} A_{i}$. Here, $t_{i}$ is the solution corresponding to $A_{l}=I_{i l}$. The velocity $\nabla_{p}$ is given in the first approximation by

$$
v_{\rho_{l}}=\frac{\partial \phi}{\partial y_{l}}=\left(I_{l j}+\frac{\partial t_{j}}{\partial y_{l}}\right) A_{j} .
$$

By integrating Eq. (17), $p_{\rho}$ is obtained:

$$
p_{p}=-\frac{d p^{(0)}}{d x_{l}} y_{l}-i \rho\left(I_{l j} y_{l}+t_{j}\right) A_{j}+\lambda,
$$

where $\lambda$ is a constant. Since $p_{p}$ is $\Omega$-periodic the following relation stands for the determination of $\mathbf{A}$ :

$$
\frac{d p^{(0)}}{d x_{l}}-i \rho A_{l}=0, \quad A_{l}=\frac{d p^{(0)}}{d x_{l}} / i \rho \text {. }
$$

The corresponding Darcy law is obtained by integrating Eq. (21) over $\Omega_{l}$ :

$$
\begin{aligned}
& \langle v\rangle_{l}=\omega^{-1}\left\langle v_{p_{l}}\right\rangle=K_{l j} \frac{d p^{(0)}}{d x_{j}}, \quad K_{l j}=\frac{n B_{l j}}{i \omega \rho}, \\
& B_{l j}=|\Omega|^{-1} \int_{\Omega_{l}}\left(I_{l j}+\frac{\partial t_{j}}{\partial y_{l}}\right) d \Omega,
\end{aligned}
$$

where $B$ is a real symmetrical tensor. As expected, the principal part, as $\omega$ becomes infinite, is imaginary, i.e., an inertial part:

$$
n \mathrm{H}_{2} / \rho \omega=\mathbf{B}^{-1} \text {. }
$$

The second approximation $\nabla_{c}$ for $\nabla_{(1)}$ is given by the boundary viscous layer, itself considered as a plane layer in first approximation. The calculation is quite classical, keeping in mind that the outward value for the $\nabla_{(1)}$ corrector is the perfect fluid solution, Eq. (21) on $\Gamma$. If $z$ denotes a dilated normal coordinate to $\Gamma$, we have ${ }^{30}$ :

$$
\nabla_{c}=-\left.\nabla_{p}\right|_{\Gamma} \exp \left[-(1+i)\left(\omega / 2 v^{\prime}\right)^{1 / 2} z\right], \quad v^{\prime}=\mu^{\prime} / \rho,
$$

and the corresponding correction $p_{c}$ for $p_{p}$ is zero. 


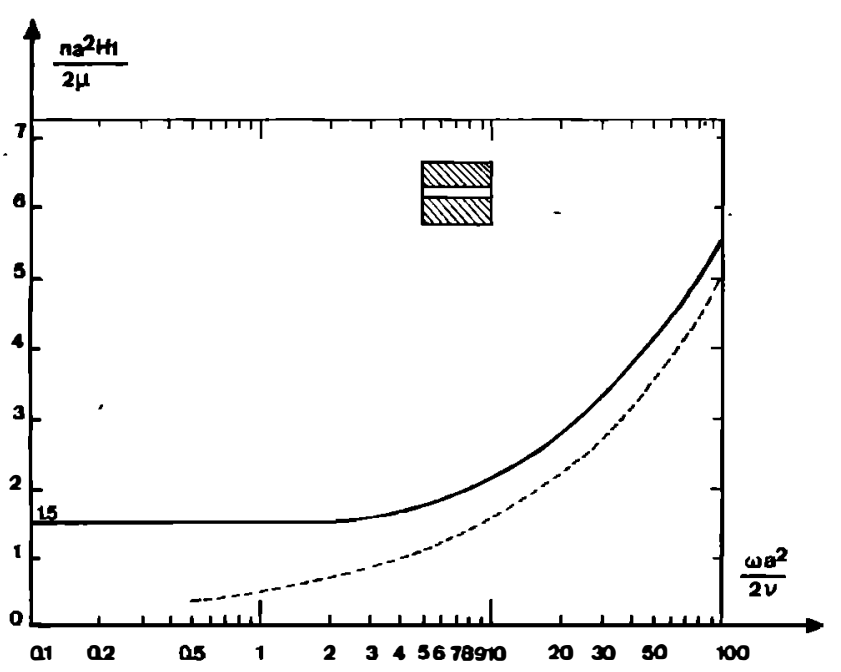

FIG. 2. Cylindrical slit: $H_{1}$ against frequency: -- asymptotic approximation $(\omega \rightarrow \infty)$.

The correction term for the Darcy law is given by integrating over $\boldsymbol{\Omega}_{l}$ :

$$
\begin{aligned}
\left\langle\omega^{-1} v_{c_{k}}\right\rangle= & -\left.n \omega^{-1}|\Omega|^{-1} \int_{\Omega} v_{p_{k}}\right|_{\Gamma} \\
& \times \exp \left[-(1+i)\left(\omega / 2 v^{\prime}\right)^{1 / 2}\right] d \Omega \\
= & -n \omega^{-1}|\Omega|^{-1} \int_{\Gamma} v_{p_{k}} d s \\
& \left.\times \int_{0}^{\infty} \exp \left[-(1+i)(\omega) / 2 v^{\prime}\right)^{1 / 2} z\right] d z \\
= & -n \omega^{-1}|\Omega|^{-1}(1+i)^{-1}\left(2 v^{\prime} / \omega\right)^{1 / 2} \\
& \times \int_{\Gamma}\left(I_{k l}+\frac{\partial t_{l}}{\partial y_{k}}\right) d s \frac{d p^{(0)}}{d x_{l}} / i \rho \\
= & n(1+i)\left(\omega^{2} \rho\right)^{-1}\left(\frac{\omega v^{\prime}}{2}\right)^{1 / 2} D_{k l} \frac{d p^{(0)}}{d x_{l}}
\end{aligned}
$$

with

$$
D_{k l}=|\Omega|^{-1} \int_{\Gamma}\left(I_{k l}+\frac{\partial t_{l}}{\partial y_{k}}\right) d s
$$

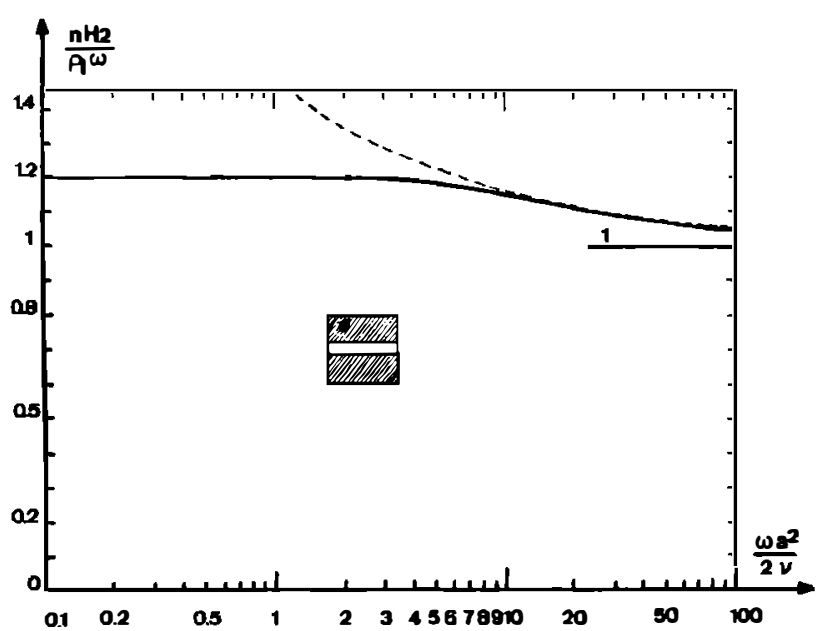

FIG. 3. Cylindrical slit: $\boldsymbol{H}_{2}$ against frequency: $\longrightarrow$ asymptotic approximation $(\omega \rightarrow \infty)$.

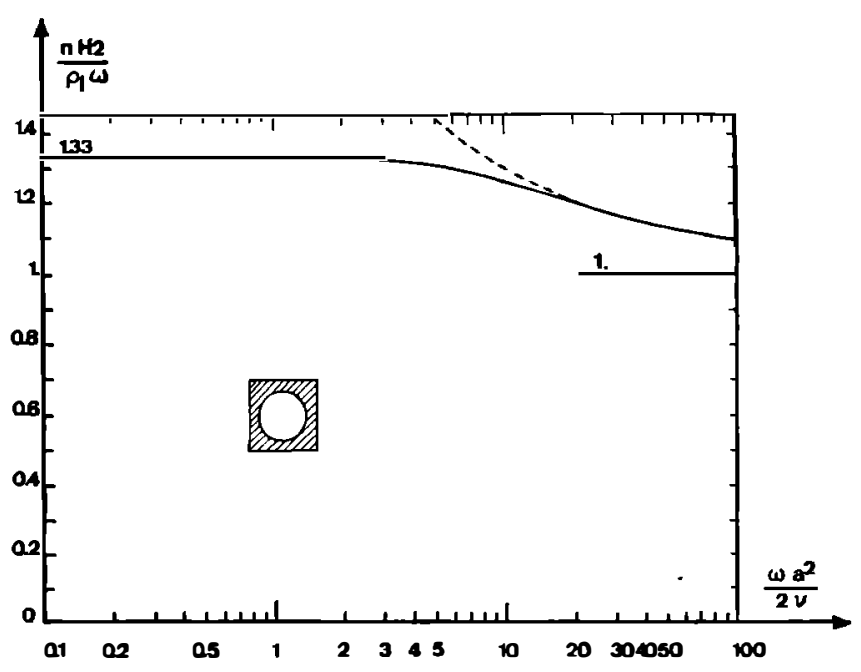

FIG. 4. Cylindrical duct: $\mathrm{H}_{2}$ against frequency: analytical result, $\cdots$ asymptotic approximation $(\omega \rightarrow \infty)$.

Finally, an approximate solution to Eqs. (14 $\dashv(16)$ leading to

$$
K_{j m}=n B_{j m} / i \omega \rho+(1+i) v^{\prime}\left(\omega / 2 v^{\prime}\right)^{1 / 2} D_{j m} / \omega^{2} \rho
$$

is obtained and, up to the second order:

$$
\begin{aligned}
H_{j m}= & n^{-1}\left(\omega \rho \mu^{\prime} / 2\right)^{1 / 2} C_{j m} \\
& +i \rho \omega n^{-1}\left[B_{j m}^{-1}+\left(v^{\prime} / 2 \omega\right)^{1 / 2}\right] C_{j m},
\end{aligned}
$$

where

$$
C_{j m}=B_{j l}^{-1} D_{l n} B_{n m}^{-1}
$$

is a shape coefficient the dimension of which is the inverse of a length. The asymptotic approximation [Eq. (22)] is quite correct for $\mathrm{H}_{2}$ since this quantity appears in the dynamic equation in the form $\mathrm{H}_{2} \omega^{-1}$. But $\mathrm{H}_{1}$ needs a third-order approximation, which is a constant, and may be introduced taking into account the curvature of the boundary layer. Finally, let us notice that the calculation of tensors $B, D$, and $C$ needs only volume or surface mean value processes from the solution of relation (20). The numerical cost is far below that of the full problem [Eq. (10)].

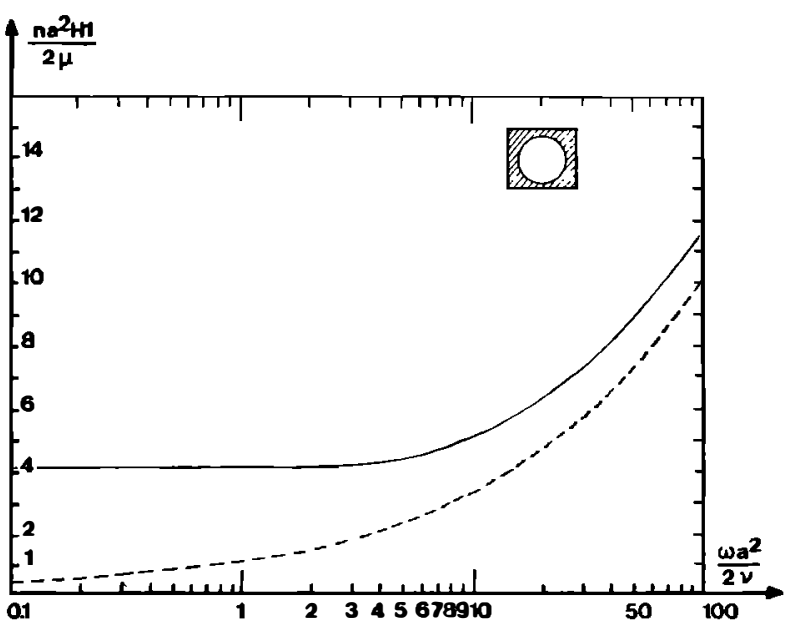

FIG. 5. Cylindrical duct: $H_{1}$ against frequency: —analytical result, ---asymptotic approximation $(\omega \rightarrow \infty)$. 

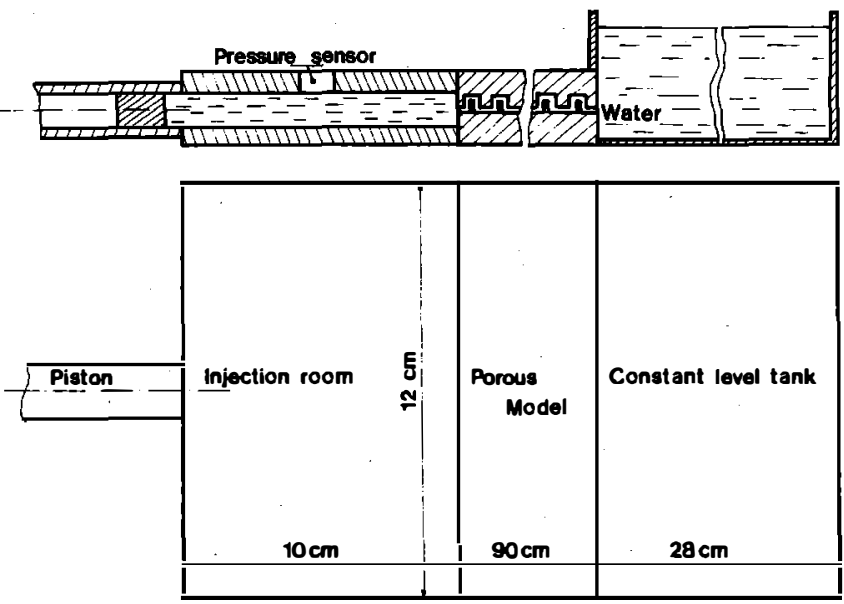

FIG. 6. Experimental setup.

To illustrate this, we have plotted in Figs. 2-5 the dimensionless values of $H_{1}$ and $H_{2}$ against the dimensionless frequency for a cylindrical slit and a circular cylinder where analytical results have been mentioned above. As expected, for all cylindrical pores we get $B_{11}=1$. In fact, this value represents the minimum value for $B_{11}^{-1}$ [see Eq. (13)]. When the tortuosity is larger, $B_{11}^{-1}$ can be found to be far greater than one (see Ref. 29 and the sequel).

\section{EXPERIMENTAL CHECKING}

The experimental setup ${ }^{29}$ is sketched in Fig. 6. A periodic porous medium undergoes a harmonic given flux at one end, a constant level reservoir ensuring a constant pressure at the other end. A low pressure membrane sensor $( \pm 33$ $\mathrm{kPa}$ ) is flush-mounted in the injection room for the determination of the macroscopic gradient pressure. The porous medium is of high tortuosity: A period is shown in Fig. 7. The $\times 2$

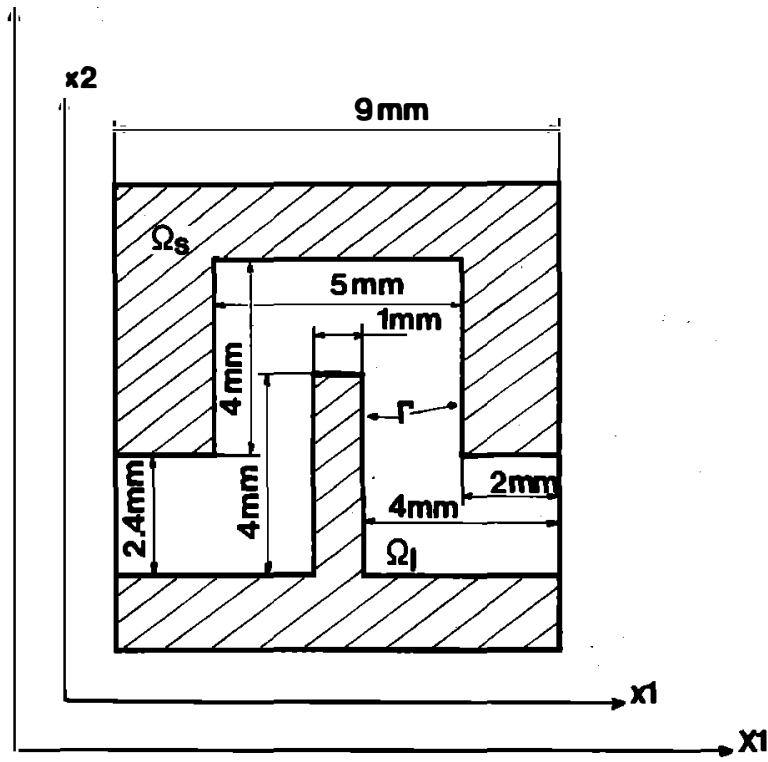

FIG. 7. A period of the porous medium.

porous model consists of a hundred periods, related to a small value for $\epsilon$. The liquid is a disaerated water. The pore size is such that the frequency range of interest, from 0.05-8 $\mathrm{Hz}$, can be produced in a mechanical manner. The model is large enough to ensure a two-dimensional behavior (this is easily verified comparing analytical results for a cylindrical slit to a rectangular cylinder) and its rigidity is such that the deformation of the porous matrix is negligible. The harmonic flux is provided by a $1-\mathrm{cm}$-diam piston with variable amplitude, with a view to satisfying the condition $\operatorname{Re}<1$. A displacement sensor with a response time corresponding to 100 $\mathrm{Hz}$ is used for the flux control. In Fig. 8 the pressure amplitude-flux amplitude ratios are plotted versus the frequency

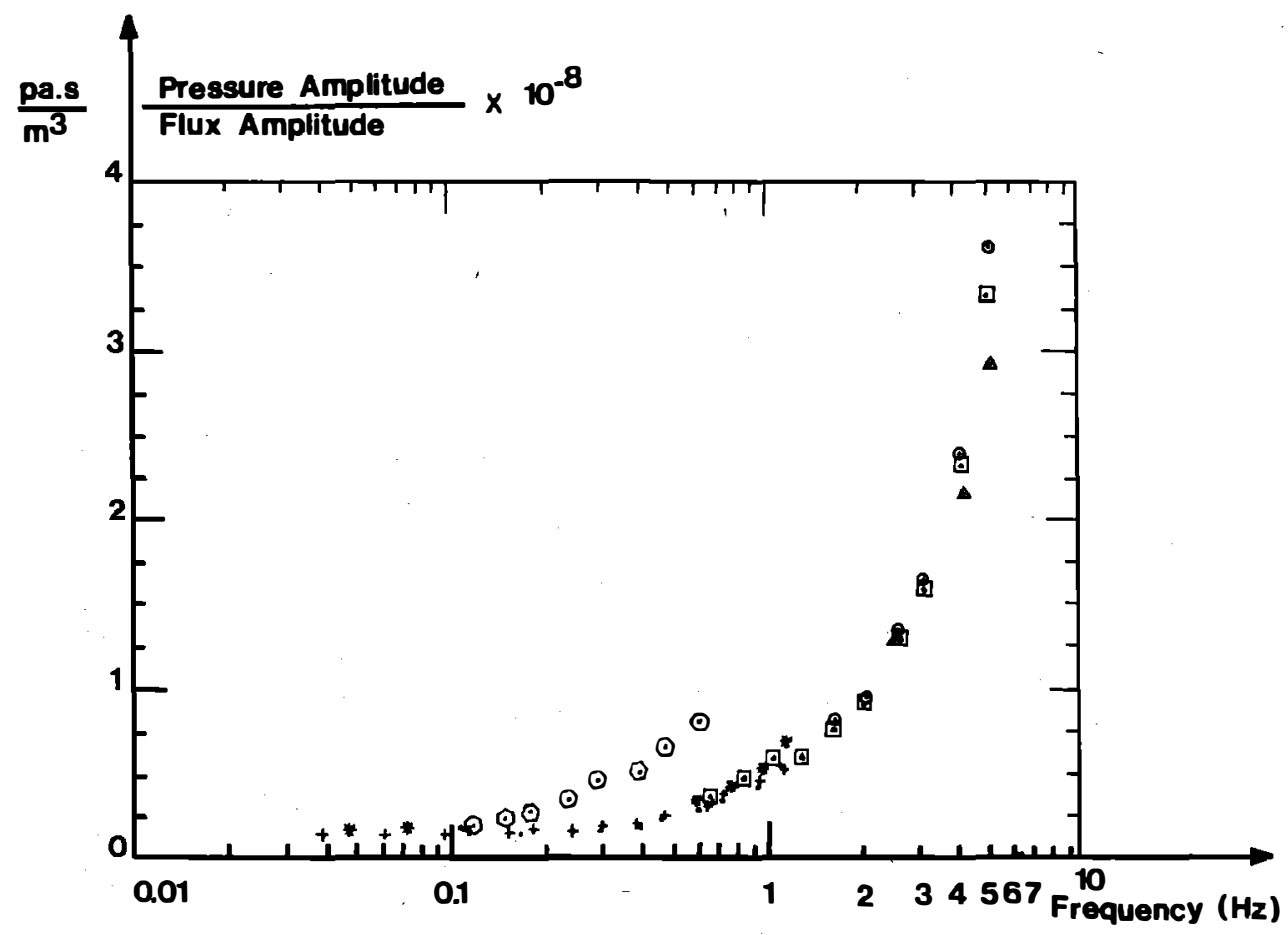

FIG. 8. Experimental data for different piston amplitudes (in $\mathrm{mm}$ ): $\odot 1.6 \mathrm{~mm}$, $.2 .2 \mathrm{~mm}, 3.4 \mathrm{~mm}, \Delta 6.3 \mathrm{~mm},+6.6$ $\mathrm{mm}, 23.8 \mathrm{~mm}, \odot 59.4 \mathrm{~mm}$. 


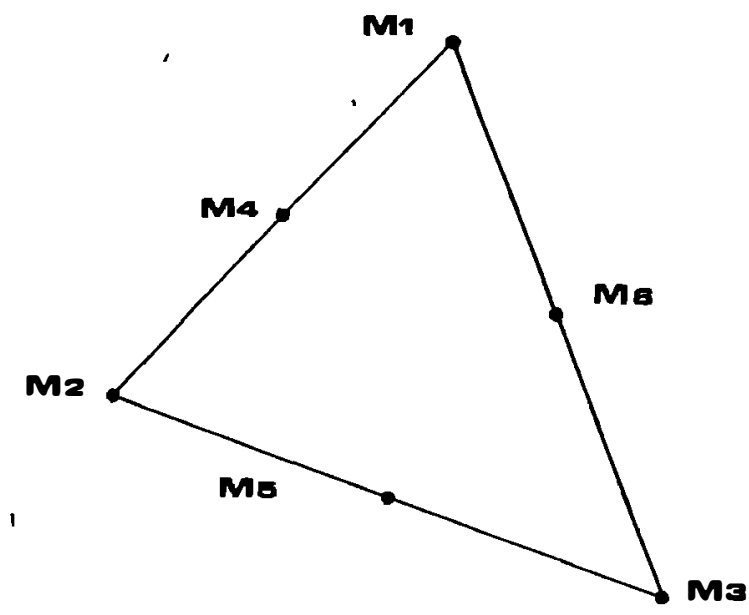

FIG. 9. Triangular element.

for different flux amplitudes. The experimental points show the apparition of nonlinearities when the frequencies increase and give the upper limits for this investigation.

The numerical computation is carried out by using formulations expressed in Eqs. (10) and (20) and a finite element method. More details are available in Refs. 29 and 31. Two meshes were considered: a rough mesh $\mathbf{A}$ and a mesh B, using the hydrodynamical streamlines of a perfect fluid. ${ }^{32}$ With these two meshes, two types of triangular elements were used.

(a) Elements with a constant complex pressure and a quadratic complex vectorial function for velocity, defined through six points $M_{i}, i=1, \ldots, 6$ (the vertices and the middle point of each edge, see Fig. 9). Let $\left(z_{1}, z_{2}, z_{3}\right)$ be the barycentric coordinates defined on an element, with $z_{1}+z_{2}+z_{3}=1$ and consider the six second-degree functions:

$$
\begin{aligned}
& \Phi_{i}(M)=z_{i}\left(2 z_{i}-1\right), \quad \text { for } \mathrm{i}=1,2,3, \\
& \Phi_{4}(M)=4 z_{1} z_{2}, \quad \Phi_{5}(M)=4 z_{2} z_{3}, \\
& \Phi_{6}(M)=4 z_{1} z_{3} .
\end{aligned}
$$

Then the velocity at a point $M$ of the element is taken as

$$
\mathbf{V}(M)=\sum_{i=1}^{6} \mathbf{V}\left(M_{i}\right) \Phi_{i}(M),
$$

where the six vectors $\mathbf{V}\left(M_{i}\right)$ are the nodal unknowns.

(b) Elements with a "bulb function" (as defined in Ref. 33) where the pressure is a complex linear function defined at the vertices and the velocity a complex vectorial function of the third degree. Consider the three functions:

$$
\Psi_{i}(M)=z_{i}, \quad i=1,2,3 .
$$

The pressure is defined by

$$
p(M)=\sum_{i=1}^{3} \Psi_{i}(M) p\left(M_{i}\right)
$$

where the $p\left(M_{i}\right)$ are the nodal unknowns for $p$ at the vertices of the element. The "bulb function" $B(M)$ is introduced by

$$
B(M)=z_{1} z_{2} z_{3}
$$

and the velocity is expressed as

$$
\mathbf{V}(M)=\sum_{i=1}^{6} \mathbf{V}\left(M_{i}\right) \Phi_{i}(M)+\beta B(M),
$$

where $\beta$ denotes a complex constant vector. Since $B(M)$ is zero valued in $M_{i}$, the $\mathbf{V}\left(M_{i}\right)$ represent the velocity values at point $M_{i}$. The introduction of this third-degree term enables a minimization of the error. ${ }^{33}$

The difficulty related to the incompressibility condition is solved with the help of Uzawa's algorithm (see Refs. 34 and 29 for the stationary Stokes problem) and the numerical process is tested on the cylindrical slit problem where analytical result is available. The experimental data for the dimensionless modulus and the phases of $H$ are plotted against the dimensionless frequency in Figs. 10 and 11 and compared to



FIG. 10. $H$ against frequency: + experimental data, - asymptotic approximation; numerical results: $\Delta$ mesh A without bulb function, $\triangle$ mesh $A$ with bulb function, $O$ mesh $B$ without bulb function, $\bigcirc$ mesh $B$ with bulb function. 


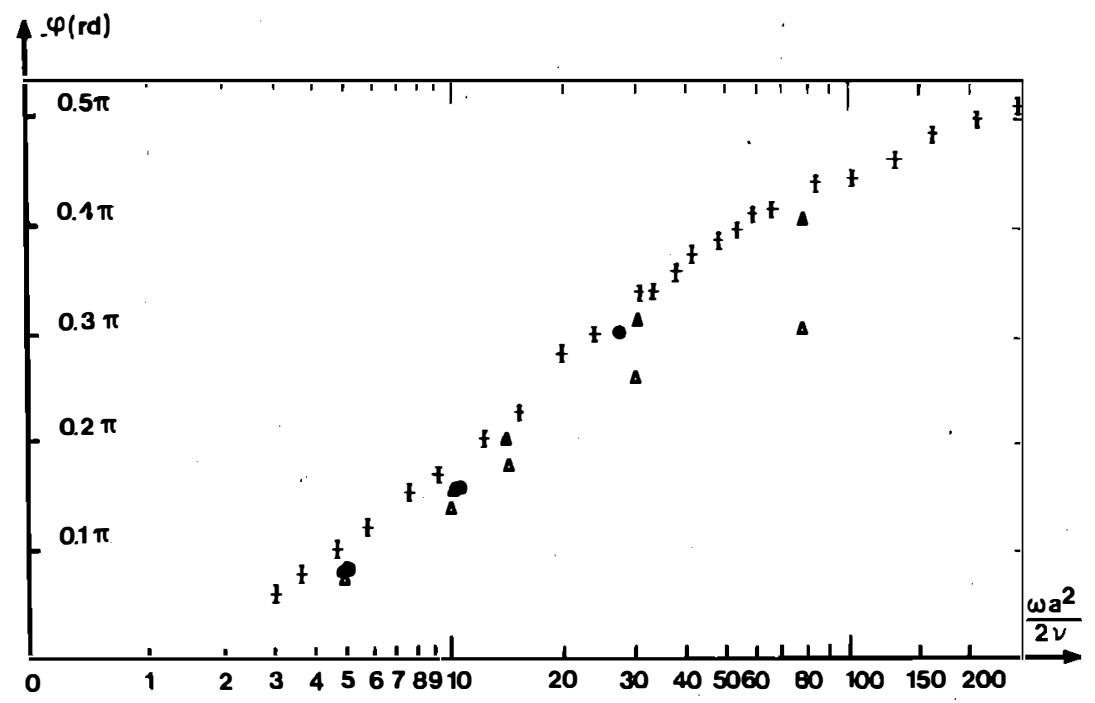

FIG. 11. Phase against frequency. Notations as in Fig. 10.

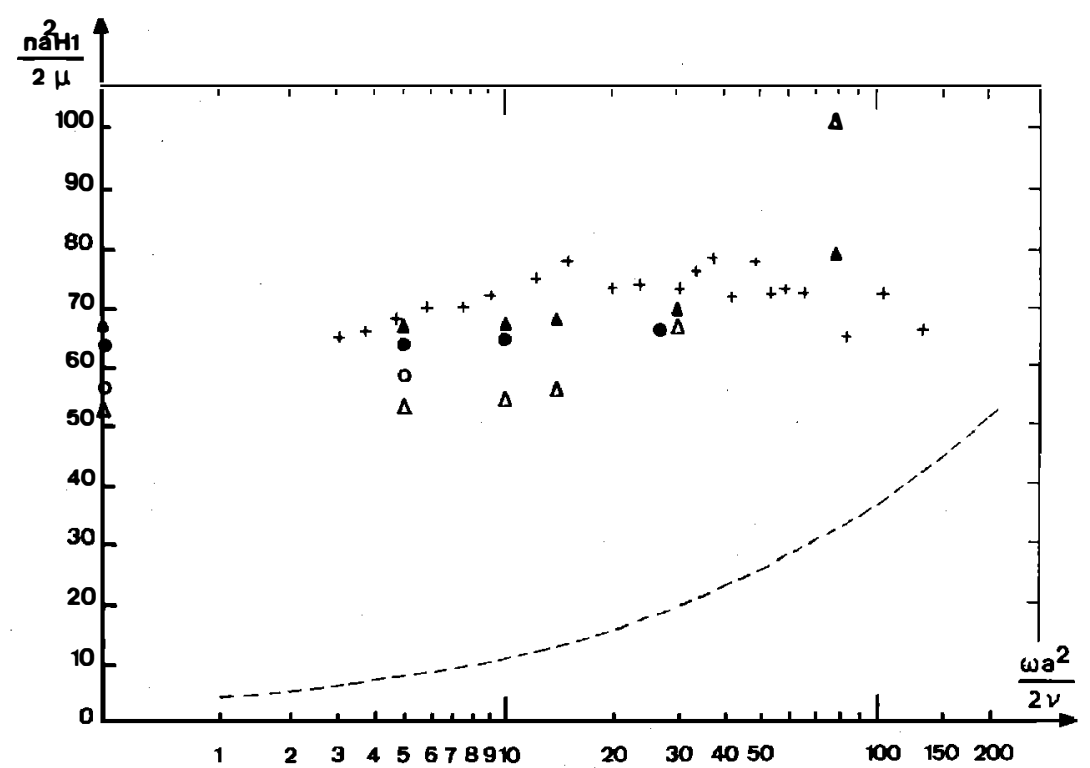

FIG. 12. $H_{1}$ against frequency. Notations as in Fig. 10.

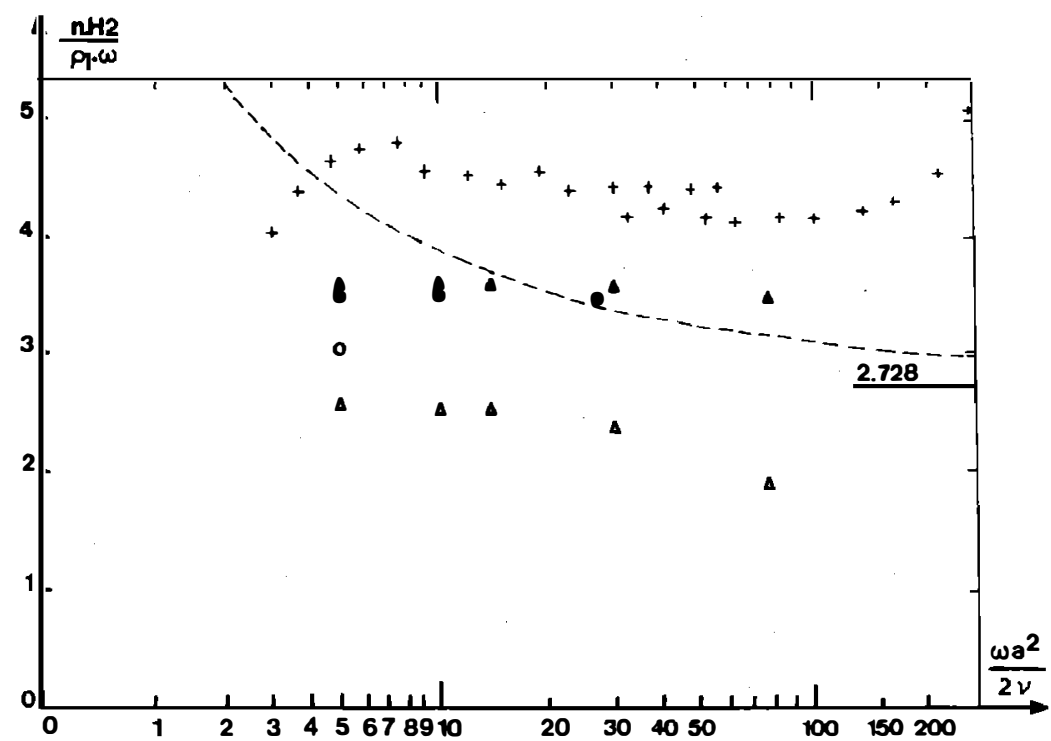

FIG. 13. $H_{2}$ against frequency. Notations as in Fig. 10. 


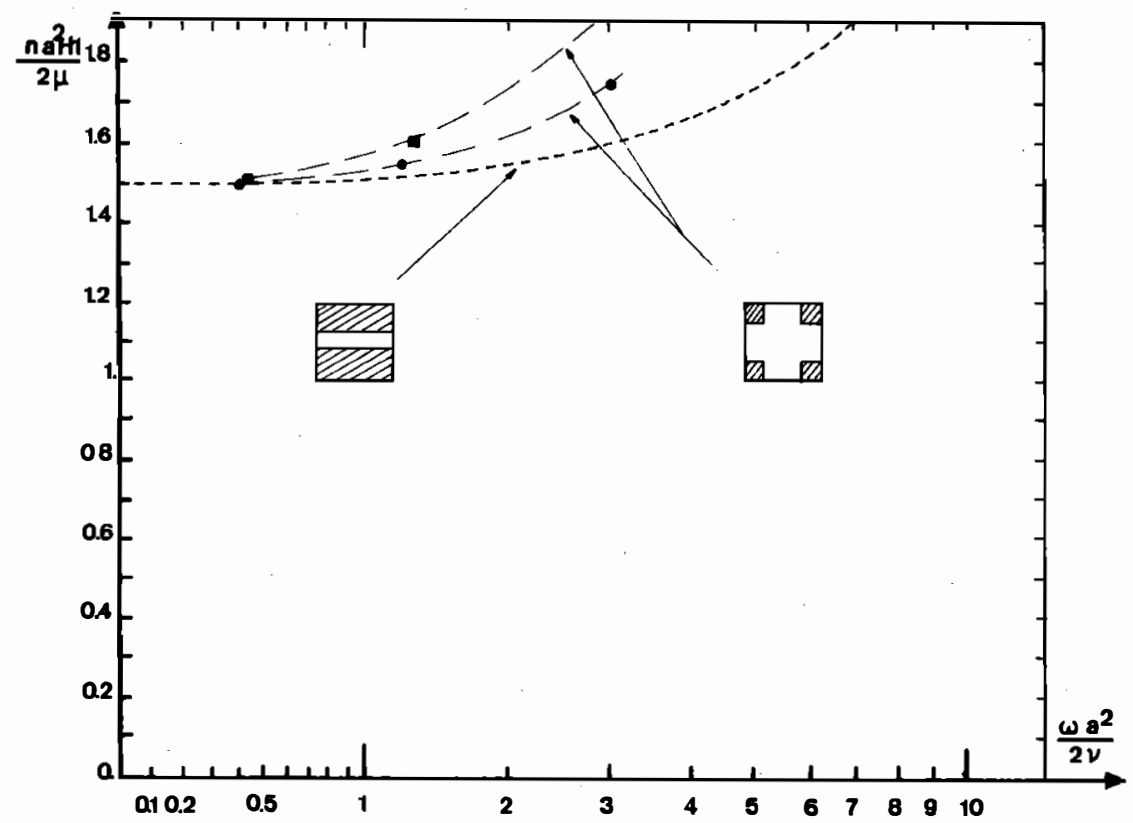

FIG. 14. Cylindrical slit (analytical result) and two slits systems (numerical result). $H_{1}$ against frequency: $\square$ without bulb function, $\bigcirc$ with bulb function.

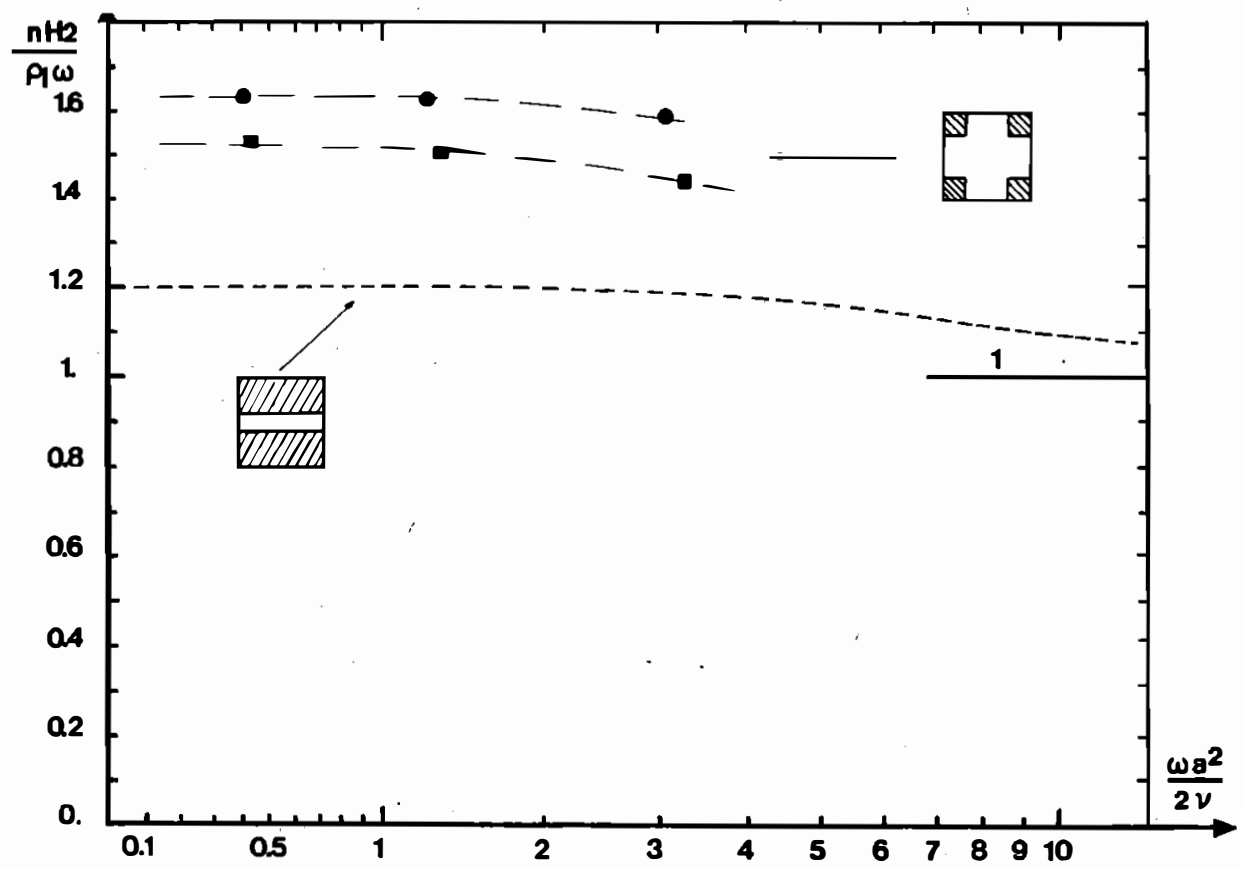

FIG. 15. Cylindrical slit (analytical result) and two slits systems (numerical result). $\mathrm{H}_{2}$ against frequency. $\square$ without bulb function, $\boldsymbol{O}$ with bulb function.

the numerical computation with the two meshes and the two types of elements. The characteristic length $a$ involved in the dimensionless frequency is $4 \cdot 10^{-3} \mathrm{~m}$. As expected, the elements with a bulb function lead to best results. An agreement is found between the experimental data and the numerical results. In a similar way Figs. 12 and 13 give the dimensionless real and imaginary parts of $H$. The major part of the discrepancy is here introduced by the behavior of the cosinus and sinus functions near zero and $\pi / 2$ values of the phase. The asymptotic behavior, as $\omega$ increases, is also sketched in Figs. 10, 12, and 13. Note that for the geometry under consideration the quantity $\mathrm{nH}_{2} / \rho \omega$ reaches the value 2.7 instead of 1 for cylindrical ducts, as $\omega$ goes to infinity.
As a final example, the case of a slit (where analytical results are available) can be compared to the case of a double system of connected perpendicular slits (numerical results following Sec. III). Partial results are plotted in Figs. 14 and 15, for the dimensionless values of $H_{1}$ and $H_{2}$ versus the dimensionless frequency. The values discrepancy is quite important: The presence of connected perpendicular ducts notably increases the inertial part and in a minor way the dissipative part. The limit of $n H_{2} / \rho \omega$, as $\omega$ goes to infinity, is 1.5.

\section{CONCLUSION}

The results of the present analysis lead us to outline the importance of the geometry, when the generalized Darcy 
coefficient is considered. Consequently, great care should be taken when using the analytical model of cylindrical ducts. A precise description of the dynamic behavior of porous saturated media needs either an experimental determination of $K(\omega)$ or a computation permitted by the periodicity.

${ }^{1}$ M. A. Biot, "Theory of propagation of elastic waves in a fluid saturated porous solid. I. Low-frequency range. II. Higher frequency range," J. Acoust. Soc. Am. 28, 168-191 (1956); "Generalized theory of acoustic propagation in porous dissipative media," J. Acoust. Soc. Am. 34, 1254 1264 (1962); "Mechanics of deformation and acoustic propagation in porous media," J. Appl. Phys. 33, 1482-1498 (1962).

${ }^{2}$ T. J. Plona, "Observation of a Second Bulk Compressional Wave in a Porous Medium at Ultrasonic Frequencies," Appl. Phys. Lett. 36, 259-261 (1980).

${ }^{3}$ D. L. Johnson, T. J. Plona, C. Scala, F. Pasierb, and H. Kojima, "Tortuosity and Acoustic Slow Waves," Phys. Rev. Lett. 49, 1840-44 (1982).

4J. G. Berryman, "Elastic waves propagation in fluid saturated porous media," J. Acoust. Soc. Am. 69, 416-424 (1981); "Elastic Waves in Fluidsaturated Porous Media," in Macrascopic Properties of Disordered Media, Locture Notes in Physics, Vol. 154 (Springer-Verlag, New York, 1981), pp. 38-50.

5J. Geertsma and D. C. Smit, "Some Aspects of Elastic Wave Propagation in Fluid Saturated Porous Solids," Geophysics 26, 169-181 (1961).

6J. P. Jones, "Effect of a uniform flow on elastic wave in a porous, saturated elastic solid," J. Acoust. Soc. Am. 34, 1172-1183 (1962).

'Y. K. Kim and H. B. Kingsbury, "Dynamic Characterization of Poroelastic Materials," Exp. Mech. 19, 252-258 (1979).

${ }^{8}$ S. R. Massel and C. C. Mei, "Transmission of Random Wind Waves Through Perforated or Porous Breakwaters," Coast. Eng. 1, 78 (1977).

${ }^{9}$ C. C. Mei and M. A. Foda, "Wave Induced Responses in a Fluid-filled Poroelastic Solid with a Free Surface-a Boundary Layer Theory," Geophys. J. R. Astron. Soc. 66, 597-631 (1981).

${ }^{10} \mathrm{D}$. Salin and Schon, "Acoustics of Water Saturated Packed Glass Spheres," J. Phys. Lett. 42, 470-480 (1981).

"A. M. Wijesinghe and H. B. Kingsbury, "On the dynamic behavior of poroelastic materials," J. Acoust. Soc. Am. 65, 90-95 (1979).

${ }^{12}$ C. H. Yew and P. N. Jogi, "Study of wave motions in fluid saturated porous rocks," J. Acoust. Soc. Am. 60, 2-8 (1976); "The Determination of Biot's Parameters for Sandstones. Part I: Static Tests," Exp. Mech. 18, 167-172 (1978).

${ }^{13}$ N. C. Dutta, "Theoretical Analysis of Observed Second Bulk Compressional Wave in a Fluid Saturated Porous Solid at Ultrasonic Frequencies," Appl. Phys. Lett. 37, 898-900 (1981).

14 L. B. Steimer and C. K. Sollitt, "Non Conservative Wave Interaction with Fixed Semi-immersed Rectangular Structures," Sixteenth Coastal Engineering Conference, Hamburg 133, 2209-2227 (1978).

15O. S. Madsen, P. Shusang, and S. A. Hanson, "Wave Transmission
Through Trapezoidal Breakwaters," Sixteenth Coastal Engineering Conference, Hamburg 129, 2140-2151 (1978).

${ }^{16}$ D. L. Johnson, "Elastodynamics of Porous Media," in Macrascopic Properties or Disordered Media, Lecture Notes in Physics, Vol. 154 (SpringerVerlag, New York, 1981), pp. 97-110.

${ }^{17}$ A. Bedford and J. D. Ingram, “A Continuum Theory of Fluid Saturated Porous Media," J. Appl. Mech. 1-7 (1971).

${ }^{18}$ A. E. Green and P. M. Naghdi, “A Dynamical Theory of Interacting Continua," Int. J. Eng. Sci. 3, 231-241 (1965).

${ }^{19} \mathrm{~L}$. Hsieh and C. H. Yew, "Wave Motions in a Fluid Saturated Porous Medium," J. Appl. Mech. 20, 873-878(1973).

${ }^{20}$ J. L. Auriault and E. Sanchez-Palencia, "Etude du Comportement Macroscopiqued'un Milieu Poreux Saturé Déformable," J. Mec. 16, 575-603 (1977).

${ }^{21}$ T. Levy, "Propagation of Waves in a Fluid-Saturated Porous Elastic Solid," Int. J: Eng. Sci. 17, 1005-1014 (1979).

${ }^{22}$ J. L. Auriault, "Dynamic Behavior of a Porous Medium Saturated by a Newtonian Fluid," Int. J. Eng. Sci. 18, 775-785 (1980).

${ }^{23} \mathrm{R}$. Burridge and J. B. Keller, "Poroelasticity equations derived from micro-structure," J. Acoust. Soc. Am. 70, 1140-1146 (1981).

24J. L. Auriault, "Homogénéisation, Application aux Milieux Poreux Saturés," Cours de DEA Mécanique, Grenoble (1980); "Homogenization. Application to Porous Saturated Media" in Two Medium Mechanics, Summer School, Gdansk, 5-9 September 1981. Publication of the Gdansk Polytechnic Institute.

${ }^{25}$ C. Avallet, "Comportement Dynamique de Milieux Poreux Saturés Déformables," Thèse de Doctorat de Spécialité, Grenoble 16 September 1981.

${ }^{26}$ J. L. Auriault and C. Avallet, “Comportement Dynamique d'un Amortisseur composé d'un Milieu Poreux Saturé, J. Mec. Theor. Appl. 1, 1-22 (1982).

${ }^{2}$ A. Bensoussan, J. L. Lions, and G. Papanicolaou, Asymptotic Analysis for Periodic Structures (North-Holland, Amsterdam, 1978).

${ }^{28}$ E. Sanchez-Palencia, Non-Homogeneous Media and Vibration Theory, Locture Notes in Physics, Vol. 127 (Springer-Verlag, Berlin, 1980), Part II.

${ }^{29} \mathrm{~L}$. Borne, “Contribution à l'Etude du Comportement Dynamique des Milieux Poreux Saturés Déformables. Etude de la Loi de Filtration Dynamique," Thèse de Docteur-Ingénieur Grenoble 29 September 1983.

${ }^{30} \mathrm{~L}$. Landau and E. Lifchitz, Mécanique des Fluides (Mir Moscow, 1971), p. 109.

${ }^{31}$ L. Bome, R. Chambon, and J. L. Auriault, "Conforming Finite Element Computations Applied to Spatially Periodic, Harmonic Navier-Stokes Problem" (to be published).

${ }^{32}$ R. Chambon, “Application de la Méthode des Eléments Finis et d'une Loi Rhéologique Incrémentale aux Calculs de Mécanique des Sols," Thèse de Docteur-Ingénieur Grenoble, 1975.

${ }^{33}$ M. Crouzeix and P. A. Raviart, "Conforming and non-conforming finite element methods for solving the stationary Stokes equations," R.A.I.R.O. Analyse Numérique R.3 (1973), pp. 33-76.

${ }^{34}$ R. Teman, Navier-Stokes Equations (North-Holland, Amsterdam, 1977). 